\title{
Beyond the bench: A conversation with Audrey Brumback
}

\author{
BY CHARLES Q. CHOI
}

3 NOVEMBER 2021

Audrey Brumback did not plan to follow in the footsteps of her father - a doctor, a scientist and the founder of the Journal of Child Neurology. She went to medical school but tried out new specialties - OB-GYN and then ophthalmology. Ultimately, though, those forays in healing were unsatisfying compared with what she discovered to be her true calling.

"It wasn't until I did neurology and then pediatrics and then child neurology that I felt my shoulders relax and had that sigh of relief," says Brumback, assistant professor of neurology and pediatrics at the University of Texas at Austin.

Brumback is now a physician-scientist who studies how the brain's circuitry is disrupted in neurodevelopmental conditions such as autism. She hopes to someday devise therapies for autism that target those circuitry differences.

She spoke with Spectrum about volunteering in Mexico, having a lab next door to her husband's and why she sometimes cries at work.

\section{Spectrum: What big question drives your research?}

Audrey Brumback: Can we localize different parts of cognition and social and emotional functioning to create targeted therapies for symptoms?

In neurology, we are trained to 'localize,' which means that the first thing we do is not to figure out what the issue is, but where the issue is. Once we know where in the nervous system something is happening, we can come up with the list of things that could be causing it. In mouse models we use a number of techniques - optogenetics (stimulating neurons with light), calcium imaging, electrode recordings - to understand where and how the brain's circuitry is disrupted in various disorders. I hope this work will one day lay the groundwork for brain stimulation in people, to help treat 


\section{Spectrum | Autism Research News}

https://staging.spectrumnews.org

problems with things like attention, memory and sensory processing.

\section{S: How did you come to focus on autism?}

AB: When I was finishing my child neurology training, I felt pretty undifferentiated. I loved all of neurology. I trained to record the electrical activity of the brain, so it would have been a natural fit for me to go into epilepsy, but I figured the field of epilepsy already has a ton of great physiologists. The field didn't really need me.

So I started thinking about the fields of neurology that could use a good electrophysiologist, and I was drawn to these conditions that are at the interface between psychiatry and neurology. Conditions like autism, where overall the structure of the brain is OK, but clearly the functioning, the physiology, is very different. I thought, "Now that's a condition where my skill set could possibly make a difference."

\section{S: Do you have any personal connection to autism?}

AB: My brother had what my parents described to me as "nonverbal learning disabilities" as a child, and it wasn't until I became a child neurologist that I realized he is probably autistic. I never knew! My brother's condition wasn't a conscious reason for my decision to specialize in autism, but it surely influenced me. As a doctor, I see a lot of kids and adults who remind me — fondly- of my brother.

\section{S: What does a typical day look like for you?}

AB: Get up at 6:30. Wake up kids at 6:50. Drop off kids at 8 . Home by 6 p.m. to relieve the babysitter. Family time from 6 p.m. to 8 p.m., then an episode of "Ted Lasso" or Stephen Colbert, then back to work till I can't keep my eyes open anymore.

During the day, I'm meeting with people on my team, hopefully writing ideas and proposals, and analyzing data. One day a week I'm in clinic taking care of kids with autism. About two to three nights per month I'm on call for the general neurology service at the Dell Children's Medical Center of Central Texas.

\section{S: What do you eat or drink while you're working?}

AB: Instant coffee.

\section{S: Do you listen to music while you write or analyze data?}

AB: I listened to the instrumental version of the "Hamilton" soundtrack while grant-writing recently. I found it really energizing. 


\section{S: You're fluent in Spanish. Has that been helpful?}

AB: Speaking Spanish has certainly helped in patient care - about half of my families are primarily Spanish-speaking.

And I have been able to spend about a week a year in Mexico as a volunteer for the Rotary Club's Proyecto Niño annual medical service mission. Child neurology is a niche field within medicine, so it was very special to me to go down to provide that expertise to people who might not otherwise have access to it. We visited the same place year after year and were able to establish relationships with patients - I am really sad I have not been able to go for the past two years because of the pandemic.

I felt we were able to do some good, and it was also just incredibly rewarding. I feel like I learned so much about how disability, risk and death are viewed in different cultures. I also learned just how different the practice of medicine is when you don't have access to technology. For neurology, CT scans and MRIs are routine parts of care in the United States, but when I was volunteering, I had to make a treatment plan based solely on a person's history and physical signs.

\section{S: What do you consider your most valuable or unusual collaboration?}

AB: My husband, MacKenzie Howard, has a lab next to mine, and it's awesome. It's been so nice to have somebody that I can talk to, bounce ideas off, get a second opinion from. It's funny when you mention to people that you work with your husband, the first reaction is, "Oh, how's that going?" as if it's definitely going to be a challenge. But it's been fantastic. We have joint lab meetings where each of our people gets feedback from the whole crew.

\section{S: In a 2019 article in Spark magazine, you talk about having imposter syndrome. Do you still struggle with that?}

AB: My imposter syndrome, that feeling of self-doubt, has decreased over time. It's partly because I'm not doing brand-new things anymore. Medical school and residencies and post-docs and being a new PI (principal investigator), those are all new experiences in which you only see the tips of everyone else's success icebergs, and you wonder how they manage when you can't. Now that I'm in my fourth year as a PI, I feel like l've got this.

\section{S: Are you active on social media?}

AB: I follow a lot of people on Twitter. It has been helpful to find out about new work I would have missed otherwise. I've been trying to amplify voices of people who are often ignored, such as by 
retweeting the Black in Neuro crew and what Black doctors, nurses and researchers are saying about the COVID-19 vaccine on The Conversation.

\section{S: Does your lab have a mascot?}

AB: We get a monthly chocolate shipment from Cocoa Runners — does that count?

\section{S: What do you do for fun?}

AB: I like to write haiku in my head when I've got a moment. I used to write a haiku a day as a diary entry.

\section{S: How do you get your news?}

AB: Doomscrolling.

\section{S: When you are not doomscrolling, what do you read?}

AB: Lots of graphic novels. My favorite series right now are "The Witch Boy" and "Sanity and Tallulah."

I also read to my kids constantly. I think reading to kids is really, really, really important, and I give all my kids in clinic a book. I like to start out the clinic visit by writing the kid's name in the book and giving it to them as a gift. Right now, it's just books I buy or bring from home, but I just got a grant from the Texas Medical Association Alliance to purchase books for kids in our clinics.

\section{S: I see you have an acting credit on the Internet Movie Database?}

AB: Yes! My high school friend Russell Barrett was in film school at New York University, so when we'd come home for holiday breaks we'd film movies. It was great fun. I think my dad must have made that IMDB entry! He was the best hype man and promoter.

\section{S: What else do you think is important for others to know?}

AB: I have depression that is super well-controlled with antidepressants. Thank God for modern medicine!

On a separate but related note, I'm not ashamed that I sometimes cry at work. It's normal to cry with strong emotions. I don't think it's healthy to hide this and wear a poker face all the time.

Cite this article: $\mathrm{https}: / /$ doi.org/10.53053/GLEK2047 